\title{
Tumores testiculares bilaterais por hiperplasia congênita de restos adrenais
}

\author{
Bilateral testicular tumors caused by congenital adrenal rest hyperplasia
}

Virginia Oliveira Fernandes', Antonio Iran Souza Barros', Ana Rosa Pinto Quidute', Ana Paula Dias Rangel Montenegro', Eveline Gadelha Pereira Fontenele', Ana Paula Abreu Martins Sales', Renan Magalhães Montenegro', Francisco Valdeci de Almeida Ferreira ${ }^{2}$, Renan Magalhães Montenegro Jr. ${ }^{1}$

${ }^{1}$ Serviço de Endocrinologia e Diabetes, Hospital Universitário Walter Cantídio, Universidade Federal do Ceará (HUWCUFC), Fortaleza, CE, Brasil ${ }^{2}$ Departamento de Patologia e Medicina Legal, UFC, Fortaleza, CE, Brasil.
Correspondência para: Renan Magalhães Montenegro Jr. Rua Professor Costa Mendes, $1.608,5^{\circ}$ andar

60416-200 - Fortaleza, CE, Brasil renanji@ufc.br

Recebido em 17/Set/2009 Aceito em 14/Nov/2009

\section{RESUMO}

Objetivos: Tumores testiculares são uma rara condição associada à hiperplasia adrenal congênita (HAC) que decorrem da hiperplasia de restos adrenais intratesticulares (HRA), raramente ocorrendo associados a neoplasias malignas. Sua diferenciação histológica com tumores de células de Leydig é muito difícil, podendo levar a orquiectomias desnecessárias. $\mathrm{O}$ objetivo deste relato foi apresentar esse dilema diagnóstico em um paciente com HAC e tumores testiculares bilaterais. Métodos: Relatou-se o caso de um paciente masculino, 16 anos, com diagnóstico de HAC desde os 3 anos de idade, que apresentava tumorações testiculares endurecidas, indolores e de crescimento lento, sendo encaminhado para orquiectomia bilateral. Resultados: Foi decidido por tratamento conservador com prednisona, havendo significativa diminuição do volume testicular e normalização dos níveis de andrógenos. Conclusão: Este caso demonstra a importância de sempre se considerar a hipótese de HRA intratesticulares no diagnóstico diferencial dos tumores testiculares. A investigação e a conduta devem ser conduzidas de maneira cautelosa para se evitar orquiectomias desnecessárias. Arq Bras Endocrinol Metab. 2009;53(8):1052-8

Descritores

Hiperplasia adrenal congênita; tumor testicular; hiperplasia de restos adrenais intratesticulares

\begin{abstract}
Objectives: Testicular tumors are a rare condition associated with congenital adrenal hyperplasia $(\mathrm{CAH})$, originated from intratesticular adrenal rest tumors, and they are rarely associated with malignant tumors. Their histological differentiation from Leydig-cell tumors is quite difficult, which would lead to inappropriate orchiectomies. Thus the objective of this report was to present this diagnostic dilemma. Methods: Reported the case of 16-yr-old boy with previous diagnosis of $\mathrm{CAH}$ with bilateral testicular enlargement who was recommended to be submitted to a bilateral orchiectomy. Results: Considering this findings, it was decided to treat conventionally with prednisone with significant reduction of testicular volume, and normalization of androgens levels. Conclusion: This case shows the importance of intratesticular adrenal rest tumors in the differential diagnosis of testicular tumors. Cautious approach during investigation and treatment are recommended to avoid inappropriate orchiectomies. Arq Bras Endocrinol Metab. 2009;53(8):1052-8
\end{abstract}

Keywords

Congenital adrenal hyperplasia; testicular tumor; intratesticular adrenal rest hyperplasia

\section{INTRODUÇÃO}

Dacientes com hiperplasia adrenal congênita (HAC) 1 apresentam um defeito na síntese do cortisol ocasionado por deficiência em graus variáveis das enzimas que participam na metabolização dos seus precursores. Con- sequentemente, ocorre secreção aumentada de ACTH hipofisário, estimulando a hiperplasia do córtex adrenal e a produção dos precursores androgênicos. Crianças com HAC podem apresentar aumento da velocidade de crescimento, avanço da idade óssea, baixa estatura final 
e puberdade precoce induzida pelo excesso de andrógenos circulantes (1).

Tumores testiculares unilaterais ou bilaterais têm sido descritos como complicação rara associada à HAC. Acometem principalmente pacientes com histórico de acompanhamento clínico inadequado e de mau controle dos níveis androgênicos com a corticoterapia (2). Tais tumores podem crescer progressivamente, adquirir características de lesões neoplásicas e causar infertilidade por comprometimento da espermatogênese e da produção de testosterona. São resultantes da hiperplasia de tecidos adrenais residuais intratesticulares - hiperplasia de restos adrenais (HRA), por efeito da estimulação crônica do ACTH (3).

Por falta de critérios histológicos e imuno-histoquímicos bem definidos, é muito difícil diferenciar essas lesões do tumor de células de Leydig (TCL), que é uma neoplasia potencialmente maligna e rara dos testículos. Esse diagnóstico é fundamental, pois a HRA e o TCL são enfermidades com prognóstico e tratamento completamente diferentes. $\mathrm{O}$ tratamento do TCL geralmente requer uma orquiectomia total, enquanto a HRA pode regredir completamente após a otimização do tratamento glicocorticoide que suprima a secreção do ACTH (4).

Assim, observando que a ocorrência de tumores testiculares em pacientes portadores de HAC constitui um dilema diagnóstico e terapêutico, além de ser uma associação pouco descrita na literatura, traz-se aqui o relato de um paciente portador de HAC, que se apresentou com volumosas tumorações testiculares bilaterais e que obteve uma surpreendente resposta terapêutica inicial à corticoterapia.

\section{RELATO DO CASO}

Paciente masculino portador de HAC diagnosticada aos 3 anos de idade, a qual se manifestou por pubarca, macrogenitossomia, hipertrofia muscular e crescimento estatural rápido observados desde o primeiro ano de vida, mas sem relato de alterações testiculares. Apresentava, nessa ocasião, estatura elevada para a idade, com desvio-padrão (DP) de $+3,9$, idade óssea (IO) avançada, de 13 anos (com idade cronológica de 3 anos) e hipertrofia muscular. As determinações laboratoriais séricas àquela época revelaram 17-hidroxiprogesterona (17-OHP): 540 ๆg/dL (valor de referência: 5-100 $\eta \mathrm{g} / \mathrm{dL}, \mathrm{RIE}$ ), cortisol basal: $8,8 \mu \mathrm{g} / \mathrm{dL}$ (valor de referência: $5,0-19,0 \mu \mathrm{g} / \mathrm{dL}, \mathrm{RIE})$, ACTH: $210 \rho \mathrm{g} / \mathrm{mL}$ (valor de referência: 10-37,0 $\mathrm{\rho g} / \mathrm{mL}$, RIE) DHEA-S:
$240 \mu \mathrm{g} / \mathrm{dL}$ (valor de referência: < $30 \mu \mathrm{g} / \mathrm{dL}, \mathrm{RIE}$ ), sódio e potássio de $136 \mathrm{mEq} / \mathrm{L}$ e $3,9 \mathrm{mEq} / \mathrm{L}$, respectivamente. Nessa ocasião, fora iniciado tratamento com prednisona $5 \mathrm{mg} /$ dia. No entanto, o paciente não aderiu ao tratamento, perdendo o seguimento após um ano e meio de acompanhamento, por condições socioeconômicas desfavoráveis.

Aos 16 anos de idade, após vários anos sem consultas, o paciente retorna ao Serviço de Endocrinologia e Diabetes do HUWC-UFC referindo crescimento progressivo dos testículos há cerca de um ano. Relatava uso irregular da prednisona e suspensão desta há aproximadamente oito meses. Havia sido avaliado por urologista em outro serviço, tendo recebido diagnóstico de neoplasia testicular bilateral e sido indicada orquiectomia bilateral.

Apresentava ao exame físico: $135,5 \mathrm{~cm}$ de estatura (DP: -5,6); $49 \mathrm{~kg}$ de peso; PA de 160 x $110 \mathrm{mmHg}$; hipertrofia muscular; testículos com volume aumentado, de consistência endurecida, com formações nodulares indolores na superfície (Figuras l e 2).

À ultrassonografia se observavam testículos aumentados de volume, ecotextura heterogênea, contornos irregulares, exibindo múltiplas formações nodulares sólidas de permeio, evidenciando aumento difuso da vascularização glandular, o testículo direito medindo $6,7 \mathrm{x}$ $6,4 \times 7,5 \mathrm{~cm}$ (volume: 167,2 $\mathrm{cm}^{3}$ ) e o esquerdo: $7,1 \mathrm{x}$ $5,7 \times 3,9 \mathrm{~cm}$ (volume: $82 \mathrm{~cm}^{3}$ ), sugestivos de lesão de natureza neoplásica.

Os exames laboratoriais nessa ocasião revelavam níveis séricos bastante elevados de testosterona, androstenediona, DHEA-S e 17OH-progesterona e níveis baixos de potássio (Tabela 1 ).

Foi realizada uma tomografia computadorizada de abdome que mostrava glândulas adrenais aumentadas de volume bilateralmente e ausência de adenomegalias retroperitoneais (Figura 3).

Foi realizada uma biópsia do testículo direito (incisional), cujo histopatológico revelava uma neoplasia formada por células poligonais de citoplasma amplo, claro a eosinofílico, com núcleo levemente pleomórfico e nucléolo evidente, dispostas em lençóis entremeados por delicado eixo fibroso, sem mitoses ou necrose, sendo compatível com TCL. A imuno-histoquímica mostrava expressão de PS100 (proteína neural), marcação focal por vimentina (células mesenquimais) e nenhum dos seguintes marcadores: EMA (antígeno de membrana epitelial), HMB45 (melanossoma), MIBl (proliferação celular) e PLAP (fosfatase alcalina placentária), achados que sugeriam o diagnóstico de TCL (Figuras 4 e 5 ). 

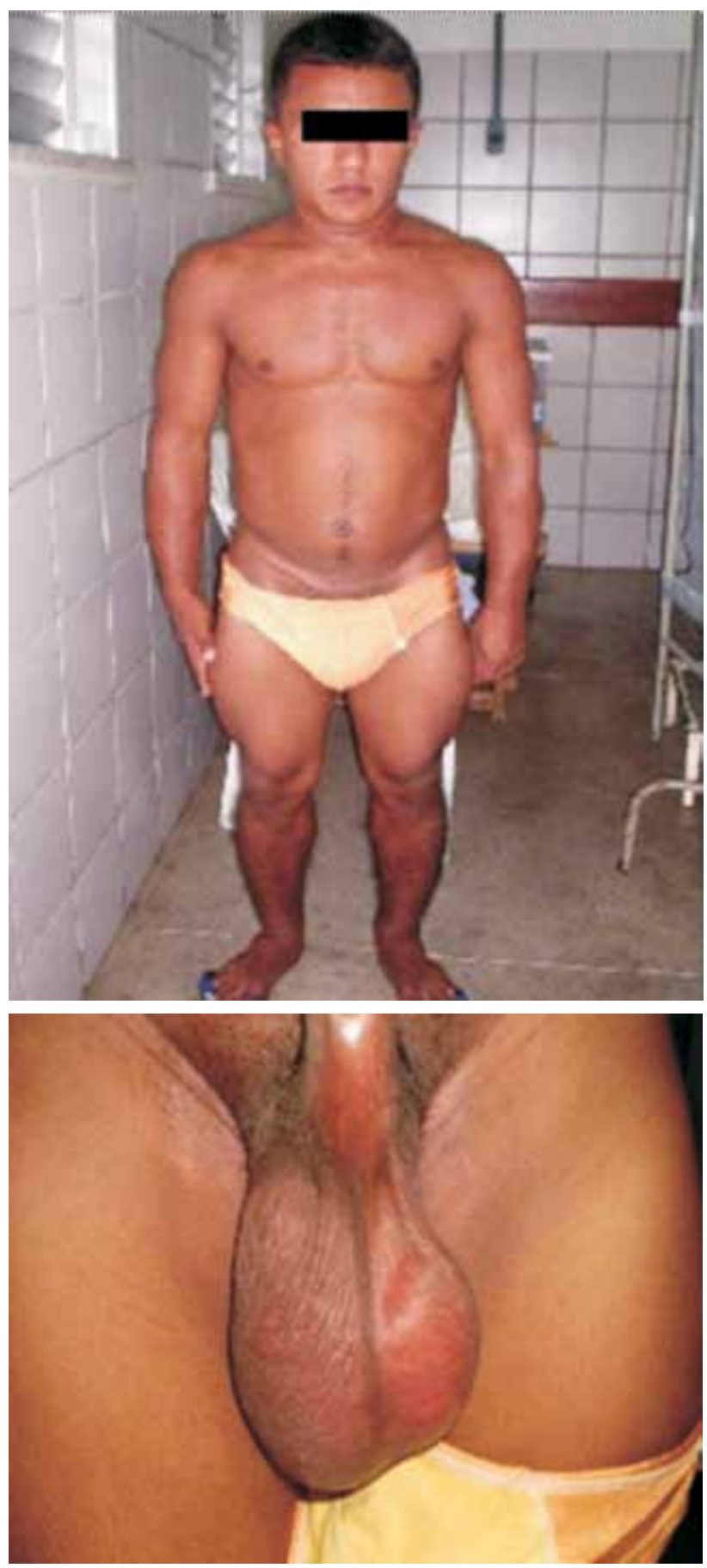

Figuras 1 e 2. Hipertrofia muscular, baixa estatura e aumento de volume testicular no paciente de 16 anos de idade em questão.

Embora os resultados do histopatológico e da imuno-histoquímica fossem compatíveis com TCL, em decorrência da reconhecida difícil diferenciação histológica entre TCL e HRA, realizou-se um teste de supressão com dexametasona - $2 \mathrm{mg} /$ dia durante cinco dias (Tabela 2), que se mostrava compatível com a HRA.

Foi realizada uma análise imuno-histoquímica complementar, havendo marcação razoável por sinaptofi-
Tabela 1. Volume testicular (VT) à ultrassonografia (US) e níveis de andrógenos e eletrólitos do paciente em questão antes e após seis meses de tratamento com prednisona

\begin{tabular}{lcc}
\hline & $\begin{array}{c}\text { Antes do } \\
\text { tratamento }\end{array}$ & $\begin{array}{c}\text { Após seis meses } \\
\text { de tratamento }\end{array}$ \\
\hline VT na US do testículo D $\left(\mathrm{cm}^{3}\right)$ & $167,2 \mathrm{~cm}^{3}$ & $33,2 \mathrm{~cm}^{3}$ \\
VT na US do testículo E $\left(\mathrm{cm}^{3}\right)$ & $82,0 \mathrm{~cm}^{3}$ & $29,2 \mathrm{~cm}^{3}$ \\
Testosterona $(\eta \mathrm{g} / \mathrm{dL})$ & 1291,0 & 406,0 \\
Androstenediona $(\eta \mathrm{g} / \mathrm{mL})$ & 189,3 & 1,6 \\
DHEA-S $(\mu \mathrm{g} / \mathrm{dL})$ & 643,0 & 68,9 \\
17OH-progesterona $(\eta \mathrm{g} / \mathrm{dL})$ & 4694,0 & - \\
11-deoxicortisol $(\eta \mathrm{g} / \mathrm{mL})$ & 1,16 & - \\
$\mathrm{LH}(\mathrm{mUl} / \mathrm{mL})$ & $<0,5$ & - \\
$\mathrm{Na}+(\mathrm{mEq} / \mathrm{L})$ & 141,0 & - \\
$\mathrm{K}+(\mathrm{mEq} / \mathrm{L})$ & 3,1 & - \\
\hline
\end{tabular}

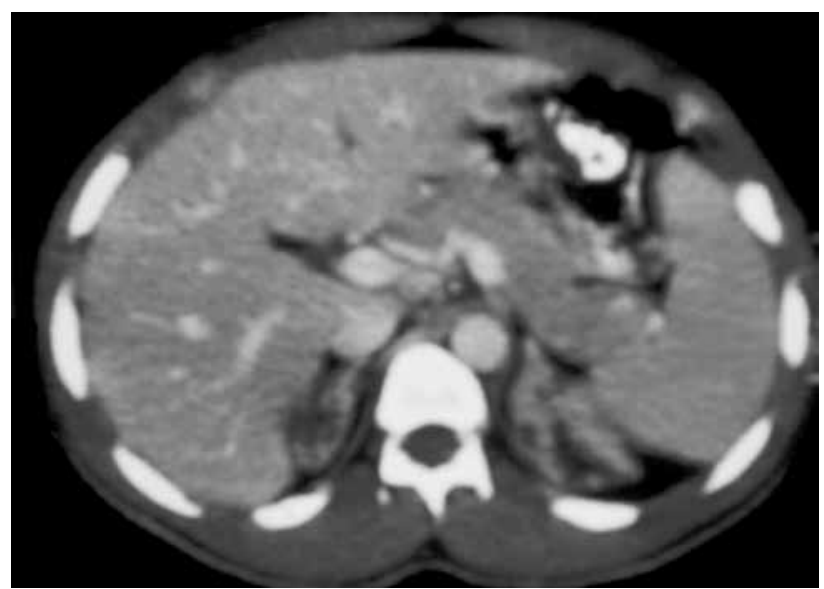

Figura 3. Glândulas adrenais aumentadas de volume bilateralmente em tomografia computadorizada de abdome antes do tratamento do paciente em questão.

sina, o que também favorecia o diagnóstico de HRA. Dessa forma, optou-se por iniciar tratamento com prednisona, $15 \mathrm{mg} /$ dia.

Após três meses, o paciente retornou apresentando redução do volume testicular bilateralmente e da consistência endurecida à palpação, sendo diminuída a dose de prednisona para $10 \mathrm{mg} /$ dia.

Após seis meses de tratamento, o paciente apresentava evidente melhora, com diminuição do volume e da consistência testicular em relação ao início do tratamento. Uma nova ultrassonografia realizada evidenciou importante redução do volume testicular: 7,6 x 2,9 x $2,9 \mathrm{~cm}$ (volume: $33,2 \mathrm{~cm}^{3}$ ) do testículo direito e $7,5 \mathrm{x}$ $3,0 \times 2,5 \mathrm{~cm}$ (volume: $29,2 \mathrm{~cm}^{3}$ ) do testículo esquerdo, ambos com ecotextura heterogênea, com nódulos hipoecoicos no parênquima medindo até $2,6 \mathrm{~cm}$. Os níveis séricos de andrógenos nessa ocasião encontravamse normais (Tabela 1 ). 


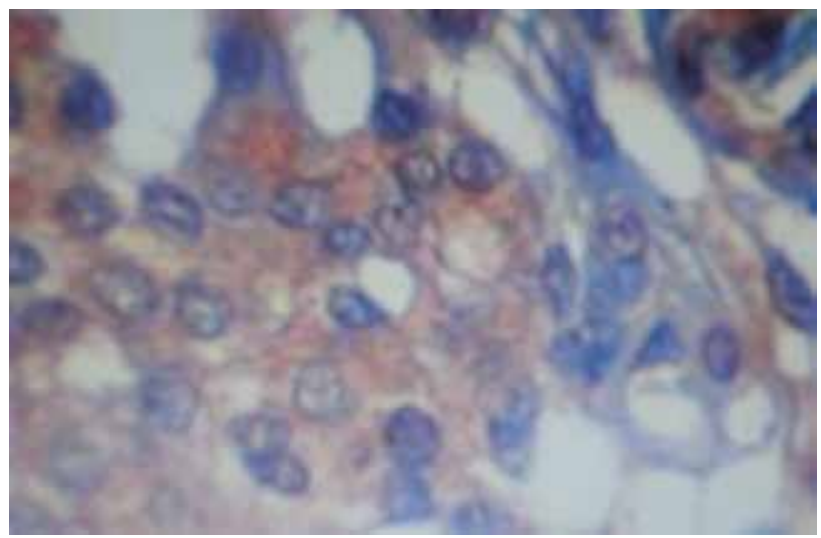

Figura 4. Imuno-histoquímica do testículo direito mostrando a expressão de PS100 (proteína neural).

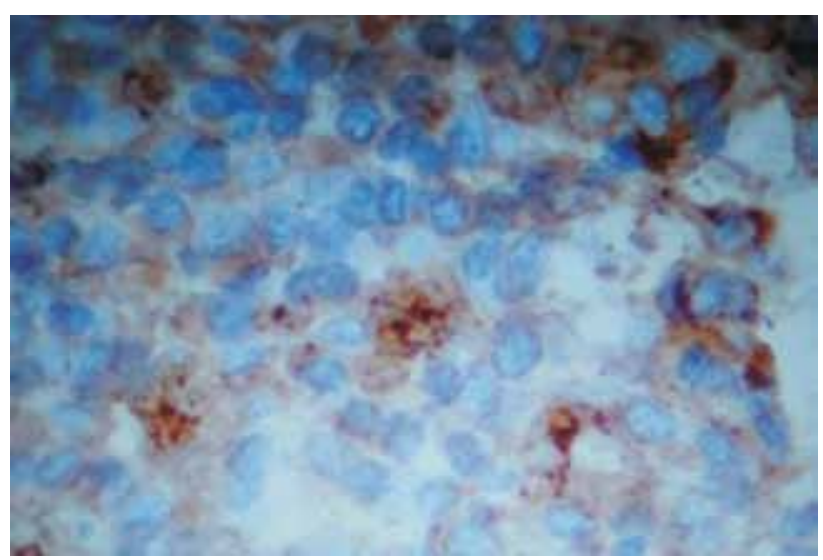

Figura 5. Imuno-histoquímica do testículo direito mostrando a marcação focal por vimentina (células mesenquimais).

Tabela 2. Níveis de andrógenos no teste de supressão com dexametasona (2 mg/dia durante cinco dias), antes do tratamento com prednisona do paciente em questão

\begin{tabular}{lcc}
\hline & Basal & Após supressão \\
\hline Testosterona $(\eta \mathrm{g} / \mathrm{L})$ & 1291,0 & 5,0 \\
Androstenediona $(\eta \mathrm{g} / \mathrm{mL})$ & 189,3 & 0,67 \\
SDHEA $(\mu \mathrm{g} / \mathrm{dL})$ & 643,0 & 3,0 \\
$170 \mathrm{H}-$ progesterona $(\eta \mathrm{g} / \mathrm{dL})$ & 4694,0 & 30,0 \\
\hline
\end{tabular}

\section{DISCUSSÃO}

Tumores testiculares em pacientes com HAC têm sido descritos há décadas, entretanto são achados clínicos raros. Podem ser unilaterais, mas tipicamente são bilaterais e sincrônicos. Acometem principalmente os pacientes inadequadamente tratados devido ao uso incorreto e irregular da corticoterapia. As lesões aparecem geralmente na segunda ou na terceira década de vida, sendo mais comuns após a puberdade (5).

As células do córtex adrenal originam-se do epitélio mesodérmico que reveste a parede abdominal posterior do embrião, mesmo tecido que origina as gônadas pri- mordiais. $\mathrm{O}$ crescimento inicial de ambas as glândulas ocorre com muita proximidade e, durante a migração das gônadas para a pelve, células adrenais podem aderir ao tecido ovariano ou alojar-se na rede testicular $(3,6,7)$. Quando restos adrenais estão presentes na rede testicular, pequenas elevações ou elevações intermitentes do ACTH e da angiotensina II (AII) podem induzir a proliferação dessas células no testículo. Sugere-se que a AII seja um importante fator na estimulação do crescimento adrenal $(8,9)$. Além disso, o controle hormonal inadequado, com elevações mais pronunciadas do ACTH, poderia acelerar esse processo. Assim, na presença de níveis elevados de ACTH, as células das suprarrenais podem ser estimuladas já intraútero. Dessa forma, tanto as concentrações quanto a duração da exposição a fatores de promoção do crescimento parecem ser importantes na patogênese do crescimento tumoral (8). Supõe-se também que o aumento do hormônio luteinizante $(\mathrm{LH})$ na puberdade pode ser um estímulo adicional para o crescimento do tumor. Como receptores LH são encontrados em HRA, isso poderia explicar o aumento da prevalência de HRA na puberdade e em pacientes pós-púberes com HAC, mesmo quando há bom controle hormonal (8). Assim, é possível que os tumores testiculares encontrados em pacientes portadores de HAC sejam resultantes da hiperplasia desse tecido adrenocortical ectópico na gônada estimulada pelos níveis elevados de ACTH e, provavelmente, também pelo LH e AII $(3,8,10)$.

A prevalência encontrada na literatura em relação à HRA em algumas séries com pequeno número de pacientes varia de 0 a $90 \%$, dependendo muito do tipo de população avaliada (idade, controle hormonal) e do método utilizado para a detecção do tumor (palpação, ultrassonografia ou ressonância nuclear magnética) $(3,11)$. Em geral, apenas tumores maiores que $2 \mathrm{~cm}$ podem ser detectados por meio da palpação, devido à sua localização dentro da rede testicular. Quando não são utilizados métodos complementares, esses tumores podem passar despercebidos (8).

Tanto a ultrassonografia quanto a ressonância nuclear magnética são bons métodos de diagnóstico como de seguimento desses tumores, mas o primeiro é preferível por ser mais barato, de mais fácil realização e por conseguir detectar restos adrenais com poucos milímetros de diâmetro $(4,12)$. Em uma série de 17 pacientes com HAC, com idade entre 16 e 40 anos, foram detectados um ou mais tumores testiculares em 16 deles por meio de ultrassonografia, sendo que somente em 
seis pacientes essas nodulações haviam sido detectadas à palpação. Cabrera e cols. (11) estudaram 30 pacientes com HAC, também adultos jovens, e detectaram nodulações testiculares em 20 pacientes usando conjuntamente os métodos de palpação e ultrassonografia. Portanto, o método de avaliação parece ser um importante fator relacionado às diferenças de prevalência de HRA em pacientes com HAC descritos na literatura. Devese, portanto, considerar a realização rotineira de ultrassonografia escrotal em pacientes portadores de HAC, principalmente após a puberdade. Não somente para a detecção, a ultrassonografia parece ser atualmente o melhor método também para seguimento clínico dessa condição, especialmente no caso de pequenos tumores não palpáveis. Essas lesões têm, geralmente, aspecto hipoecogênico nas fases iniciais e hiperecogênico nas lesões mais avançadas em consequência da fibrose (8).

As características ultrassonográficas encontradas no caso (testículos aumentados de volume, formações nodulares hipoecogênicas de dimensões variadas, limites imprecisos e multifocais, com aumento da vascularização) foram semelhantes aos achados de HRA descritos na literatura. Contudo, estes não são suficientes para diferenciar tal condição benigna dos tumores malignos, assim como sua origem adrenal $(12,13)$. A hipoecogenicidade é também encontrada em lesões neoplásicas como seminomas, mas essa característica isolada não define o diagnóstico (14).

É também difícil a diferenciação histológica entre a HRA e o TCL, se não houver correlação clínica e laboratorial adequada (5). Entretanto, Bock e cols. (15) demonstraram que há diferenças significativas em alguns critérios histológicos, estabelecendo que maior pleomorfismo nuclear, baixo índice mitótico, extensa fibrose, pigmentação abundante por lipofucsina, positividade para sinaptofisina, metaplasia adiposa e ausência de cristais de Reinke favorecem o diagnóstico de HRA.

No caso descrito, não foram evidenciados cristais de Reinke, nem mitoses ou necrose. Havia pleomorfismo nuclear e pequena quantidade de fibrose, características que favoreciam o quadro de HRA, porém, o aspecto poligonal das células, de citoplasma amplo e eosinofílico, era indistinguível do que se observa no TCL. Entretanto, a marcação razoável por sinaptofisina encontrada no caso favorecia o diagnóstico de HRA.

A presença de tecido adrenal no testículo pode ser evidenciada por meio de dosagens hormonais na veia espermática através de cateterização. Utilizando esse método, Combes-Moukhovsky e cols. (13) demonstra- ram uma concentração significativamente aumentada de compostos androgênicos $11 \beta$-hidroxilados (21-deoxicorticosterona, 21 -deoxicortisol e 11- $\beta$-hidroxi- $\Delta 4$ androstenediona) na veia espermática em relação à circulação periférica. A enzima 11- $\beta$-hidroxilase, que sintetiza os compostos citados acima a partir de seus precursores, é exclusiva das células do córtex adrenal. Assim, a existência de gradiente elevado na veia espermática é suficiente para confirmar a presença de tecido adrenocortical funcionante nos testículos. No presente caso, não foi possível a utilização desse método.

A HRA pode causar infertilidade, sendo descritas elevadas prevalências de anormalidades no espermograma, com comprometimento da qualidade do sêmen nesses pacientes (3). Tem sido sugerido que a infertilidade poderia ser causada diretamente pelo tumor interferindo na função gonadal por efeito de massa ou por alteração nos pulsos de secreção hormonal no eixo hipotálamo-hipófise-gônada (3). Alguns autores encontraram associação entre perda da função gonadal dependente da quantidade e dimensões dos nódulos testiculares na ultrassonografia (11). A localização central do tumor perto do mediastino testicular, causando compressão dos túbulos seminíferos, poderia levar à azoospermia obstrutiva e a danos irreversíveis ao tecido testicular adjacente (8). Um recente estudo demonstrou uma diminuição de diâmetro tubular e um grau variável de fibrose peritubular e hialinização tubular na biópsia testicular, além de clínica de infertilidade em sete pacientes com HAC e HRA bilateral de longa data (16). Além disso, foi observada diminuição acentuada do número de células germinativas em todos os pacientes (8). Associados aos efeitos mecânicos, os tumores também podem exercer um efeito parácrino sobre o tecido circundante, causando uma produção excessiva de estrógeno, resultante da aromatização dos andrógenos adrenais e comprometendo a secreção de LH e a função das células de Leydig (3). Esses esteroides produzidos pelas células tumorais podem ser tóxicos para as células de Leydig e para as células germinativas (17). Contudo, há vários relatos de reversão da infertilidade após regressão das massas testiculares com a corticoterapia em casos tratados mais precocemente $(4,18)$. A realização periódica de espermograma nesses casos é importante para o seguimento clínico.

Para a decisão terapêutica, os achados ultrassonográficos e as dosagens plasmáticas de $\mathrm{ACTH}$, renina e andrógenos devem ser considerados. Pacientes sem fibrose podem ser tratados com sucesso por meio do aumento da dose de glicocorticoides. A intensificação 
da corticoterapia pode levar à redução do tumor pela supressão do ACTH, melhorando, assim, a função testicular. Contudo, deve-se salientar que a elevação das doses de glicocorticoides pode trazer graves efeitos colaterais, e alguns pacientes podem não tolerar seu uso (19-21). Além disso, é possível que esse tratamento só proporcione uma melhora temporária do efeito de massa, pois a redução da dose pode levar a um novo crescimento tumoral. Apesar disso, a otimização do uso de glicocorticoides é fundamental, especialmente em pacientes com controle hormonal inadequado. No futuro, novos tipos de glicocorticoides, como a hidrocortisona de liberação lenta ou os inibidores seletivos do ACTH, poderão ajudar a suprimir o ACTH de forma mais eficaz, sem o risco de eventos adversos. O tratamento com mineralocorticoides também deve ser otimizado quando necessário em razão do efeito da AII sobre o crescimento tumoral (8).

Em estágios mais avançados, o aumento da dose de glicocorticoides provavelmente já não é eficaz em diminuir o tamanho do tumor, mas a remoção de parte da lesão pode prevenir danos adicionais ao testículo. Em virtude do caráter benigno desses tumores, cirurgias poupadoras de parte do testículo foram propostas para o tratamento de HRA nesse estágio. Alguns autores sugerem a realização de cirurgia conservadora (enucleação) para os nódulos testiculares que persistem após corticoterapia prolongada, visando maximizar o potencial de fertilidade desses pacientes (22). Walker e cols. (22) realizaram esse procedimento em três pacientes, não havendo recorrência do tumor. Tiryaki e cols. (23) relataram dois pacientes com HAC tratados também de forma efetiva com o mesma abordagem. Contudo, nenhum desses estudos relatou informações sobre a função hipófise-gonadal antes ou depois da cirurgia. Em outro estudo, não foi observada melhora da disfunção gonadal nos pacientes com HRA de longa data com tal procedimento, sugerindo ser decorrente a dano irreversível do tecido testicular (19). Além disso, lesões adicionais pela própria cirurgia não podem ser excluídas. Assim, nessa fase mais avançada de comprometimento, a única indicação para a cirurgia é o alívio da dor e do desconforto causado pela HRA (8). No caso de pacientes inférteis, a biópsia testicular pode ser útil para avaliar a viabilidade e a qualidade do parênquima testicular residual (16).

A decisão terapêutica do caso aqui relatado foi tomada baseada na história clínica prévia e nos achados bioquímicos de HAC. A indicação de orquiectomia neste caso demonstra a importância do conhecimento dos aspectos diferenciais dessas entidades, fundamentais para um correto diagnóstico. Na literatura, há relatos de casos semelhantes submetidos à orquiectomia desnecessária, cujo seguimento clínico posterior confirmou se tratar de HRA e não de TCL $(4,18)$. O TCL é uma forma rara de neoplasia testicular, representando apenas cerca de $1 \%$ a $3 \%$ dos tumores do testículo. Geralmente acomete pacientes de 20 a 60 anos, mas pode aparecer em adolescentes. Cerca de $10 \%$ a $20 \%$ são malignos e acarretam metástases nos dois primeiros anos após o diagnóstico, sendo usualmente resistentes à radioterapia e à quimioterapia (25). Não obstante, é importante ressaltar que o diagnóstico de HAC não exclui a possibilidade da sua coexistência com neoplasias malignas (24).

No presente caso houve uma resposta significativa após seis meses de tratamento com glicocorticoide (prednisona, 10 a $15 \mathrm{mg} /$ dia), evidenciada por importante redução do volume testicular à ultrassonografia e normalização dos níveis de andrógenos, reforçando o diagnóstico de tumor por HRA. Não obstante, apesar da regressão plena do volume testicular, a ultrassonografia ainda evidenciava persistência de lesões nodulares hipoecogênicas no parênquima, embora com dimensões menores. Esses achados são concordantes com a literatura. Karnak e cols. (26) acompanharam um paciente com HRA bilateral por 16 meses, sob terapia supressiva com prednisolona $10 \mathrm{mg} /$ dia, e observaram também grande redução do volume testicular e melhora da consistência, mas os achados ultrassonográficos de nódulos hipoecoicos persistiram. Por outro lado, há vários relatos de evolução com normalização do volume testicular e desaparecimento de nódulos, com o seguimento a longo prazo de tumores testiculares por HRA. Cunnah e cols. (4) relataram o desaparecimento completo da massa testicular e reversão da infertilidade após uso prolongado de dexametasona, em paciente submetido à orquiectomia contralateral desnecessariamente.

Em conclusão, o diagnóstico de hiperplasia de restos adrenais intratesticulares sempre deve ser considerado no diagnóstico diferencial dos tumores testiculares, principalmente em pacientes com hiperplasia adrenal congênita ou achados clínicos compatíveis. A investigação e a conduta devem ser conduzidas de maneira cautelosa para evitar orquiectomias desnecessárias.

Declaração: os autores declaram não haver conflitos de interesse científico neste estudo. 


\section{REFERÊNCIAS}

1. Speiser PW, White PC. Review articles: congenital adrenal hyperplasia. N Engl J Med. 2003;349:776-88.

2. Kirkland RT, Kirkland JL, Keenan BS, Bongiovanni AM, Rosenberg HS, Clayton GW. Bilateral testicular tumors in congenital adrenal hyperplasia. J Clin Endocrinol Metab. 1977;44(2):369-78.

3. Stikkelbroeck NM, Otten BJ, Pasic A, Jager GJ, Sweep CGJ, Noordam $\mathrm{K}$, et al. High prevalence of testicular adrenal rest tumors, impaired spermatogenesis, and Leydig cell failure in adolescent and adult males with congenital adrenal hyperplasia. J Clin Endocrinol Metab. 2001;86(12):5721-28.

4. Cunnah D, Perry L, Dacie JA, Grant DB, Lowe DG, Savage MO, et al. Bilateral testicular tumours in congenital adrenal hyperplasia: a continuing diagnostic and therapeutic dilemma. Clin Endocrinol (Oxf). 1989;30:141-7.

5. Srikanth $M$, West $B$, Ishitani $M$, Isaacs $H J$, Applebaum $H$, Costin G. Benign testicular tumors in children with congenital adrenal hyperplasia. J Pediatr Surg. 1992;27(5):639-41.

6. Mesinao S, Jaffe RB. Developmental and functional biology of the primate fetal adrenal cortex. Endocr Revi. 1997;18:378-403.

7. Fujieda K, Tajima T. Molecular basis of adrenal insufficiency. Pediatr Res. 2005;57:62R-69R.

8. Claahsen-Van der Grinten HL, Otten BJ, Stikkelbroeck MM, Sweep FC, Hermus AR. Testicular adrenal rest tumours in congenital adrenal hyperplasia. Best Pract Res Clin Endocrinol Metab. 2009;23(2):209-20.

9. Fallo F, Pezzi V, Barzon L, Mulatero P, Veglio F, Sonino N, et al. Quantitative assessment of CYP11B1 and CYP11B2 expression in aldosterone-producing adenomas. Eur $\mathrm{J}$ Endocrinol. 2002;147:795-802.

10. Benvenga S, Smedile G, Lo GF, Trimachi F. Testicular adrenal rests: evidence for luteinizing hormone receptors and for distinct types of testicular nodules differing for their autonomization. Eur J Endocrinol. 1999;141:231-7.

11. Cabrera MS, Vogiatzi MG, New MI. Long-term outcome in adult males with classic congenital adrenal hyperplasia. J Clin Endocrinol Metab. 2001;86:3070-8.

12. Stikkelbroeck NM, Suliman HM, Otten BJ, Hermus AR, Blickiman JG, Jager GJ. Testicular adrenal rest tumours in postpubertal males with congenital adrenal hyperplasia: sonographic and MR features. Eur Radiol. 2003;13:1597-663.

13. Combes-Moukhovsky ME, Kottler ML, Valensi P, Boudou P, Sibony M, Attalil J. Gonadal and adrenal catheterization during adrenal suppression and gonadal stimulation in a patient with bilateral testicular tumours and congenital adrenal hyperplasia. J Clin Endocrinol Metab. 1994;79:1390-4.
14. Loehrer P, Ahlering TE, Pollack A. Testicular cancer. In: Pazdur R, Coia LR, Hoskins WJ, Wagman LD, editors. Cancer management: a multidisciplinary approach. 8th edition. Georgia: FA Davis Company; 2004. p. 383-402.

15. Bock BJ, Cheville JC. Diagnostic histologic features of testicular tumor of adrenogenital syndrome: differentiation from Leydig cell tumor. Unites States and Canadian Academy of Pathology International Academy. Annual meeting. 2000;25-31.

16. Claahsen-Van der Grinten HL, Otten BJ, Hermus AR, Sweep. FC, Hulsbergen-van de Kaa CA et al. Testicular adrenal rest tumours in patients with congenital adrenal hyperplasia can cause severe testicular damage. Fertil Steril. 2008;89:597-601.

17. Murphy $\mathrm{H}$, George $\mathrm{C}$, de Kretser D, Judd S. Successful treatment with ICSI of infertility caused by azoospermia with adrenal rests in the testes. Hum Reprod. 2001;16:263-7.

18. San Miguel Fraile P, Fernandez Fernandez G, Meijide Rico F, Anton Badiola I, Ortiz-Rey JA, Alvarez Alvarez C, et al. Hiperplasia de restos adrenales en el testículo: una causa infrecuente de infertilidad masculina. Actas Urol Esp. 2003;27(3):234-9.

19. Claahsen-van der Grinten HL, Sweep FC, Blickman JG, Hermus AR, Oten BJ. Prevalence of testicular adrenal rest tumours in male children with congenital adrenal hyperplasia due to 21-hydroxylase deficiency. Eur J Endocrinol. 2007;57:339-44.

20. Giacaglia LR, Mendonca BB, Madureira G, Melo kf, Suslik CA, Arnhold IJ, et al. Adrenal nodules in patients with congenital adrenal hyperplasia due to 21-hydroxylase deficiency: regression after adequate hormonal control. J Pediatr Endocrinol Metab. 2001;14:415-9.

21. Stikkelbroeck NML, Hermus ARMM, Suliman HM, Jager GJ, Otten $\mathrm{BJ}$, et al. Asymptomatic testicular adrenal rest tumours in adolescent and adult males with congenital adrenal hyperplasia: basal and follow-up investigation after 2,6 years. J Pediatr Endocrinol Metab. 2004;17:6645-53.

22. Walker BR, Skoog SJ, Winslow BH, Canning DA, Tank ES. Testis sparing surgery for steroid unresponsive testicular tumours of the adrenogenital syndrome. J Urol. 1997;157:1460-3.

23. Tiryaki T, Aycan Z, Huecumenglu S, Atayurt H. Testis sparing surgery for steroid unresponsive testicular tumors of the congenital adrenal hyperplasia. Pediatr Surg Int. 2005;21:853-5.

24. Davis JM, Woodroof J, Sadasivan R, Stephensr. Case report: congenital adrenal hyperplasia and malignant Leydig cell tumor. Am J Med Sci. 1995;309:63-5.

25. Odabas Ö, Dilek FH, Avanoglu H, Atilla MK, Yilmaz Y, Aydins S. Leydig cell tumor of the testis. Eastern J Med. 1998;3(2):78-9.

26. Karnak I, Senocak ME, Gögüs $S$, Büyükpamukçu N, Hiçsönmez A. Testicular enlargement in patientes with 11-hydroxylase deficiency. J Pediatr Surg. 1997;32(5):756-8. 